\title{
Aktuelle Betrachtung der Tonsillektomie und Tonsillotomie
}

Katharina Geißler, Orlando Guntinas-Lichius

\begin{abstract}
Tonsillenoperationen werden bei Kindern häufig vorgenommen, und es können, wenn auch selten, erhebliche Komplikationen auftreten. Der Nutzen der Tonsillotomie und der Tonsillektomie muss deshalb systematisch evidenzbasiert analysiert werden. Auch mit der Einführung der Tonsillotomie als Regelleistung ist die Diskussion, welche Operation zu bevorzugen ist, nicht abgeschlossen. Es ist offen, ob eine Tonsillotomie gegenüber einer Tonsillektomie zur Behandlung einer rezidivierenden akuten Tonsillitis eventuell unterlegen ist.
\end{abstract}

\section{LERNZIEL}

Dieser Beitrag soll die Leser, insbesondere Kolleginnen und Kollegen in der HNO-Facharztweiterbildung, in die Lage versetzen, Patienten und Angehörige gut über das Für und Wider der Tonsillenchirurgie beraten zu können.

\section{Einleitung}

\section{Bedeutung der Tonsillenchirurgie}

Operationen an der Tonsilla palatina gehören zu den häufigsten Operationen in der HNO-Heilkunde, vor allem bei Kindern. Die Diskussion über optimale chirurgische Verfahren beschäftigt die HNO-Heilkunde spätestens seit der Veröffentlichung der Bertelsmann Stiftung „Faktencheck Gesundheit: Entfernung der Gaumenmandeln bei Kindern und Jugendlichen" [1] im Jahr 2013 bei zu hoher, nicht medizinisch begründbarer regionaler Variation der Anzahl der Tonsillenoperationen in Deutschland. Bereits davor war in Deutschland eine ständig steigende Zahl von Tonsillotomien zur Behandlung der Tonsillenhyperplasie mit oberer Atemwegsobstruktion bei Kleinkindern mit guten Behandlungserfolgen zu beobachten. Beides zusammen forcierte den Wunsch nach besseren Qualitätssicherungsverfahren zur Indikationsstellung und eröffnete die Debatte, wann eine Tonsillektomie und wann eine Tonsillotomie indiziert sei. Ein wichtiger Schritt war die Veröffentlichung der S2k-Leitlinie „Therapie entzündlicher Erkrankungen der Gaumenmandeln - Tonsillitis“ (Nr. 17/024) der Arbeitsgemeinschaft der Wissenschaftlichen Medizinischen Fachgesellschaften (AWMF) unter Federführung der Deutschen Gesellschaft für HalsNasen-Ohren-Heilkunde, Kopf-Hals-Chirurgie im Jahr 2015 [2]. Die Leitlinie versuchte erstmals in Deutschland Empfehlungen für die Tonsillenchirurgie auszusprechen.
Das Institut für Qualität und Wirtschaftlichkeit im Gesundheitswesen (IQWiG) hat dann im Auftrag des Gemeinsamen Bundesausschusses (G-BA) untersucht, ob die Tonsillotomie Vorteile gegenüber der Tonsillektomie bietet. Der IQWiG-Bericht (N15-11) wurde 2017 veröffentlicht (www.iqwig.de/de/projekte-ergebnisse/projekte/ nichtmedikamentoese-verfahren/n15-11-tonsillotomiebei-rezidivierender-akuter-tonsillitis-und-bei-hyperplasieder-tonsillen.7133.html). Im Wesentlichen wurden kurzfristige Vorteile für die Tonsillotomie erkannt, aber der langfristige Nutzen und Schaden sei unklar. 2019 wurde vom G-BA als Folge des Berichts die Tonsillotomie für die Behandlung der Hyperplasie der Tonsilla palatina in den Katalog für das ambulante Operieren aufgenommen. Andererseits wurde konstatiert, dass der Nutzen zur Behandlung einer rezidivierenden akuten Tonsillitis unklar sei und eine Erprobungsstudie zur Aufklärung ausgeschrieben werde. Diese Erprobungsstudie wird derzeit vorbereitet. Parallel zu dem IQWiG-Verfahren wurde vom G-BA die Tonsillenoperation als mengenrelevanter Eingriff eingeordnet und 2018 ein Zweitmeinungsverfahren erlassen. Danach erhielten die Patienten das formalisierte Recht, bei einer Indikationsstellung und Operationsempfehlung zunächst noch eine zweite ärztliche Meinung einzuholen (www.g-ba.de/presse/pressemitteilungen/778/).

Vor dem Hintergrund der zahlreichen regulatorischen Änderungen ist es sicher gut, dass nunmehr die Arbeit zur Aktualisierung der AWMF-Leitlinie zur Tonsillitis begonnen wurde. Die Laryngo-Rhino-Otologie hat zuletzt im Jahr 2016 in der Rubrik „Facharztwissen HNO“ einen ausführlichen Beitrag zu Operationen im Rachen publiziert [3]. Der vorliegende Artikel möchte die Leser der Laryngo-Rhino-Otologie und insbesondere die Kolleginnen und Kollegen in der HNO-Facharztweiterbildung auf den aktuellen Stand bringen. Eine Beschreibung der Operationstechniken findet sich in dem Beitrag von 2016 und wird hier nicht wiederholt. 

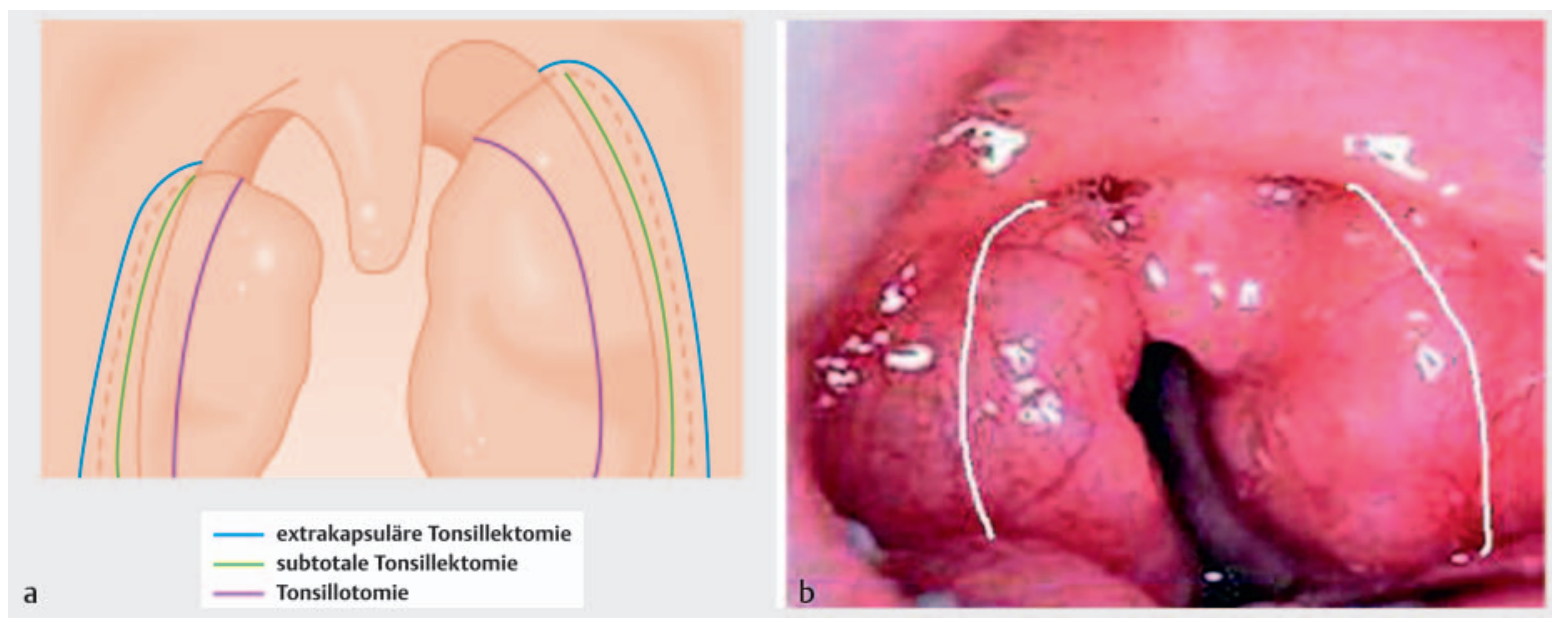

- Abb. 1 Definition des Ausmaßes der Operation bei Tonsillektomie und Tonsillotomie. a Während das Ausmaß durch die Kapsel bei der Tonsillektomie eindeutig definiert ist, ist dies bei der Tonsillotomie nicht definiert. Wie in der Zeichnung zu sehen, wird die Tonsillotomie häufig im Niveau der Gaumenbögen vorgenommen. Falls darüber hinaus nach lateral intrakapsulär operiert wird, verwendet man z. B. den Begriff subtotale Tonsillektomie. Quelle: Amrhein P, Di Dio D, Koitschev A et al. Tonsillektomie und Tonsillotomie im Wandel - Ist die Tonsillektomie bei Kindern noch zeitgemäß? Pädiatrie up2date 2019; 14 (03): 215-224 DOI: 10.1055/a-0720-1722. b Intraoperativer Situs. Nur der die Gaumenbögen überragende Teil (weiß markiert) soll laut Autoren bei der Tonsillotomie entfernt werden. Aber entspricht die weiße Linie diesem Anteil? - Dies macht deutlich, wie schwierig eine Standardisierung sein kann. Quelle: Gronau S, Fischer Y. Die Tonsillotomie. Laryngorhinootologie 2005; 84 (9): 685-690 DOI: 10.1055/s-2005-861043.

\section{Definition}

Eigentlich erscheint die Definition sehr einfach. Bei der Tonsillektomie (OPS-2020-Code: 5-281.0) wird zur vollständigen Entfernung der Tonsilla palatina eine extrakapsuläre Resektion, also eine vollständige Entfernung des Organs unter Mitnahme der Tonsillenkapsel vorgenommen. Jede Art von Teilresektion ist dann eine Tonsillotomie (OPS-2020-Code: 5-281.5), bei der dann intrakapsulär, also unter Erhalt der Kapsel, operiert wird.

Insbesondere im angloamerikanischen Raum, wo der Begriff der Tonsillotomie seltener verwendet wird, finden sich Ausdrücke wie partielle, subtotale oder intrakapsuläre Tonsillektomie. In der AWMF-Leitlinie Tonsillitis wird eine Klassifikation vorgeschlagen, über deren Nutzung im Alltag wenig bekannt ist: Wenn nur Tonsillengewebe medial der Gaumenbögen abgetragen wird, wird von einer Klasse-I-Tonsillotomie gesprochen, wird weiter nach lateral unter Erhalt der Tonsillenkapsel operiert, von einer Klasse-II-Tonsillotomie. Einen Konsens über die Unterschiede zwischen den Begriffen partielle, subtotale oder intrakapsuläre Tonsillektomie gibt es nicht ( $\vee$ Abb. 1).

\section{Merke}

Tonsillektomie $=$ vollständige chirurgische Entfernung der Tonsilla palatina

Tonsillotomie $=$ Teilentfernung der Tonsilla palatina

\section{Historische Entwicklung}

Ausführliche Beschreibungen der Historie der Tonsillenchirurgie finden sich an anderer Stelle [4, 5]. Die ersten Beschreibungen von Tonsillenentfernungen findet man bereits in der Antike. Teile der Gaumenmandeln wurden zunächst mit dem Finger oder der Schere entfernt. Die Tonsillenchirurgie begann also mit Verfahren zur Teilentfernung. Im 19. Jahrhundert kam die Teilentfernung mit Tonsillotomen, also Tonsillenschnürern, auf, vor allem mit „kalten“, später auch mit „heißen“ Schlingen, und zwar mit galvanokaustischen Glühschlingen. Die Tonsillektomie galt dagegen als technisch schwierig, und die Gefahr der lebensbedrohlichen Nachblutung war bekannt.

Bis Anfang des 20. Jahrhunderts war die Teilentfernung ohne Entfernung der Tonsillenkapsel Standard. Erst mit Aufkommen der Möglichkeit der Vollnarkose setzte dann eine Diskussion um mögliche Vorteile der Tonsillektomie ein, insbesondere bei entzündlichen Erkrankungen der Tonsille. Es mehrten sich die Beobachtungen (im Vorantibiotikazeitalter und im Zeitalter vor der evidenzbasierten Medizin), dass nach Tonsillotomie bei Patienten mit der Anamnese einer rezidivierenden Tonsillitis häufiger erneute Tonsillitiden (selten Abszesse) auftreten könnten. Letztendlich gingen nach dem 2. Weltkrieg die Tonsillotomien zurück und die Tonsillektomien nahmen zu.

Ein erneuter Umschwung geht auf die umfangreichen Fallserien aus der Wendezeit von Scherer et al. am damaligen Klinikum Benjamin Franklin der Freien Universität Berlin zurück. Zwischen 1989 und 2002 wurden dort 1374 Tonsillotomien mit dem $\mathrm{CO}_{2}$-Laser vorgenommen [6]. Seitdem, also schon weit vor der Einführung der S2k-Leitlinie in Deutschland, sind ein kontinuierlicher Rückgang der Tonsillektomie und eine Zunahme der Ton- 


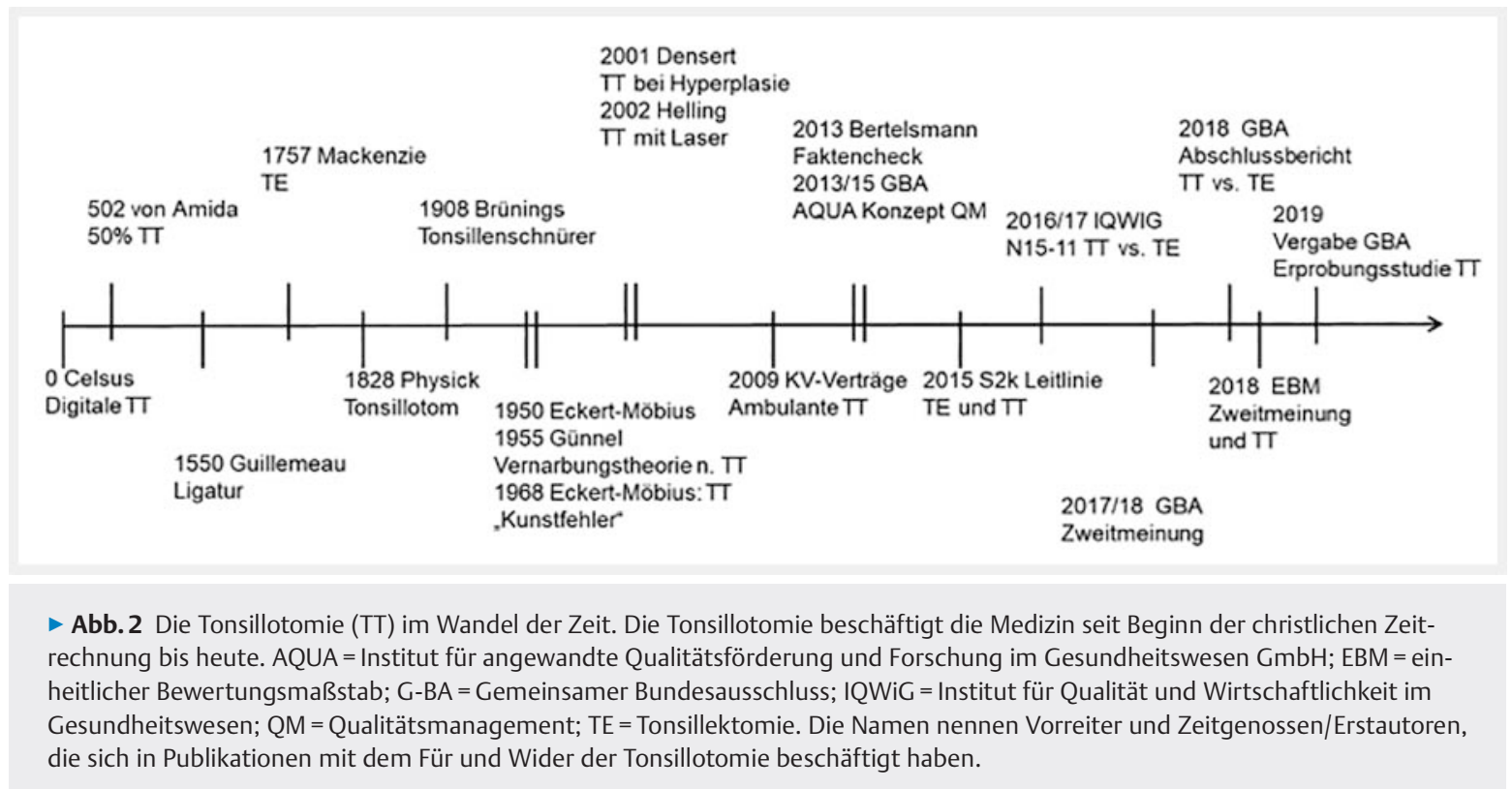

sillotomie zu beobachten [7]. Genau genommen ist seit spätestens den 1980er-Jahren nicht nur in Deutschland, sondern auch in anderen westlichen Ländern ein Rückgang der Anzahl der jährlichen Tonsillektomien zu beobachten [8]. Bereits seit dieser Zeit wird eine Debatte über die Indikation zur Tonsillektomie geführt, und die Diskussion ist noch lange nicht beendet.

\section{Merke}

In der Medizingeschichte war die Tonsillotomie weit vor der Tonsillektomie als medizinisches Behandlungsverfahren verbreitet, wurde nach dem 2 . Weltkrieg von der Tonsillektomie verdrängt und erlebte eine Wiederentdeckung in Deutschland um die Wendezeit, zunächst als Verfahren zur Behandlung der krankhaften Hyperplasie der Gaumenmandeln ( $\triangleright$ Abb. 2).

\section{Indikation}

\section{Tonsillektomie}

Wichtige klassische Indikationen zur Tonsillektomie - die zum Teil auf dem Prüfstand stehen - sind die folgenden Erkrankungen: rezidivierende akute Tonsillitis, Peritonsillarabszess, Retrotonsillarabszess, tonsillärer Fokus bei entzündlicher Grunderkrankung, Schlaf-Apnoe-Syndrom und Tumorverdacht. In Bezug auf die rezidivierende akute Tonsillitis formuliert die aktuelle S2k-Leitlinie Tonsillitis, die weiter unten noch genauer betrachtet wird, genauere Empfehlungen: Eine Tonsillektomie sei eine therapeutische Option, wenn der Patient mindestens 6 Episoden einer ärztlich diagnostizierten und mit Antibiotika therapierten eitrigen Tonsillitis in den letzten 12 Monaten vor Vorstellung erlitten hat.

\section{Tonsillotomie}

Nach dem Wiederaufkommen der Tonsillotomie wurde das Verfahren im Wesentlichen zur Behandlung der Tonsillenhyperplasie bei Kleinkindern eingesetzt, die bedingt durch die Hyperplasie der Tonsillen an Mundatmung, Schnarchen, Atemwegsobstruktion, Schlafstörungen, Schluckstörungen und Schlaf-Apnoe-Syndrom litten. Tonsillitiden in der Patientenanamnese galten lange als Kontraindikation für die Tonsillotomie. In der S2k-Leitlinie Tonsillitis wurde dies dann nicht mehr aufrechterhalten: Tonsillitiden stellten fortan keine Kontraindikation mehr dar, sondern sind seitdem ggf. sogar die Indikation. Ebenso wird keine Alterslimitierung empfohlen. Eine Tonsillotomie könne auch bei älteren Kindern und Erwachsenen zur Behandlung einer rezidivierenden akuten Tonsillitis vorgenommen werden. Dabei wird für die Indikationsstellung zur Tonsillotomie dieselbe quantitative Definition anhand der Tonsillitis-Episoden angewandt wie weiter oben für die Tonsillektomie beschrieben. Letztendlich wird in der Leitlinie die Tonsillotomie als gleichwertige Behandlungsmethode zur Behandlung der rezidivierenden akuten Tonsillitis betrachtet.

Die diskutierten Vorteile und Nachteile der Tonsillotomie versus Tonsillektomie sind in $\mathbf{T a b} \mathbf{1}$ zusammengefasst.

\section{ÜBERBLICK}

Die wichtigsten Indikationen

Tonsillektomie:

- rezidivierende akute Tonsillitis

- Peritonsillarabszess, Retrotonsillarabszess 
- Tab.1 Gegenüberstellung der diskutierten Vorteile vs. Nachteile von Tonsillotomie und Tonsillektomie.

\begin{tabular}{|c|c|c|}
\hline & Tonsillotomie & Tonsillektomie \\
\hline Vorteile & $\begin{array}{l}\text { - Komplikationsrate ist niedriger } \\
\text { - Postoperative Schmerzen sind geringer } \\
\text { - kann bei einem Teil der Patienten ambulant vorge- } \\
\text { nommen werden }\end{array}$ & $\begin{array}{l}\text { - Tonsillitiden können danach nicht mehr auftreten } \\
\text { - gut standardisiertes Verfahren } \\
\text { - kann für alle Indikationen zur Tonsillenchirurgie } \\
\text { eingesetzt werden }\end{array}$ \\
\hline Nachteile & $\begin{array}{l}\text { - erneute Operation an den Gaumenmandeln wegen } \\
\text { wiederkehrender Beschwerden ist möglich } \\
\text { - Effektivität zur Behandlung der rezidivierenden akuten } \\
\text { Tonsillitis ist nicht ausreichend untersucht } \\
\text { - Ausmaß der Teilresektion ist nicht standardisiert }\end{array}$ & $\begin{array}{l}\text { - Komplikationsrate ist höher } \\
\text { - postoperative Schmerzen sind höher } \\
\text { - Auswirkungen der kompletten Entfernung dieses } \\
\text { sekundären Immunorgans sind nicht ausreichend } \\
\text { untersucht }\end{array}$ \\
\hline
\end{tabular}

- tonsillärer Fokus bei entzündlicher Grunderkrankung

- Schlaf-Apnoe-Syndrom

- Tumorverdacht

Tonsillotomie:

- krankhafte Hyperplasie der Gaumentonsillen im Kindesalter

- rezidivierende akute Tonsillitis (laut Leitlinie, nicht ausreichend geprüft laut IQWiG)

\section{Studien, Leitlinien und regulatorische Änderungen}

\section{Faktencheck Gesundheit: Entfernung der Gau- menmandeln bei Kindern und Jugendlichen}

Im Auftrag der Bertelsmann Stiftung hat das Institut für Gesundheits- und Sozialforschung (IGES) auf Basis der Grunddaten der Krankenhäuser und derer Qualitätsberichte, der Bevölkerungsstatistik sowie im Wesentlichen der Daten des Statistischen Bundesamts über die Fallpauschalen-bezogene Krankenhausstatistik die Tonsillektomien in deutschen Krankenhäusern zwischen 2007 und 2010 untersucht. Detailliert wurde das Jahr 2010 ausgewertet [1]. Es zeigten sich erhebliche regionale Unterschiede in der Häufigkeit der durchgeführten Tonsillektomien. Auf Kreisebene zeigte sich zudem eine Spannweite von 10-116 Tonsillektomien je 10000 Kinder und Jugendliche. Die Autoren konnten keine wissenschaftliche Begründung für die Unterschiede finden. Die Variationen seien vielmehr Ausdruck eines uneinheitlichen Entscheidungsverhaltens bei der Indikationsstellung. Eine Leitlinie könne hier hilfreich sein. Auch wurde bereits angeregt, bei gegebener Indikation gegebenenfalls die Tonsillektomie durch eine Tonsillotomie zu ersetzen.
In der Presse wurde die Studie vielfach diskutiert mit Aussagen wie „Mandel-OPs bei Kindern - ein Roulette-Spiel“ (Der Spiegel: http://www.spiegel.de/gesundheit/diagnose/ kinder-und-jugendliche-wohnort-entscheidet-ueber-man delentfernung-a-897438.html). Wichtig zur sachlichen Einordnung des Faktenchecks ist, dass aus der Analyse keine Schlussfolgerungen über die Versorgungsqualität und die optimale Indikationsstellung für eine Tonsillenoperation abgeleitet werden können. Und natürlich steht die HNO-Heilkunde in Deutschland mit der Frage einer konsentierten Indikationsstellung für ein operatives Verfahren nicht allein da. Bereits 1938 versuchte man die regionale Variabilität der Tonsillektomie in Großbritannien zu verstehen [9, 10].

Auch viele andere, häufig durchgeführte Operationen zeigen eine erhebliche geografische Variabilität. Unterschiede in den Schweregraden der Erkrankung, der diagnostischen Abklärung oder Patientenverhalten können in der Regel nur einen kleinen Teil der regionalen Variabilität erklären. Vielmehr scheint es, dass chirurgische Variabilität ganz allgemein in erster Linie Ausdruck der individuellen Entscheidungsfindung des Arztes und der Einbeziehung des Patientenwunsches in die Entscheidung ist [11]. Auch nach Einführen der Leitlinie ist zumindest bis 2017 immer noch eine bedeutsame regionale Variabilität in der Anzahl der Tonsillektomien in Deutschland festzustellen [7]. Untersuchungen zur Leitlinien-Adhärenz liegen nicht vor.

\section{Merke}

In Deutschland besteht weiterhin eine bedeutsame regionale Variabilität in der Durchführung der Tonsillektomie. Für die Tonsillotomie ist dies auch zu vermuten.

\section{AWMF-Leitlinie zur Therapie entzündlicher Erkrankungen der Gaumenmandeln - Tonsillitis}

Die Leitlinie hat initial zu erheblichen Diskussionen unter den HNO-Ärzten geführt. Daher ist eine genauere Betrachtung wichtig. Zunächst einmal handelt es sich um eine 
S2k-Leitlinie und nicht um eine S3-Leitlinie. Es erfolgte also eine strukturierte Konsensusfindung durch eine repräsentative, interdisziplinäre Expertengruppe mit nachvollziehbarem methodischem Vorgehen, aber keine Angabe von Evidenz- und Empfehlungsgraden, da keine systematische Aufbereitung der Evidenz zugrunde lag. Bei der Leitlinie konnte für alle Empfehlungen ein $100 \%$ iger Konsens erreicht werden. Das Kapitel zur Tonsillenchirurgie stellt das umstrittenste Kapitel der Leitlinie dar.

Grundlage zur Bewertung der Tonsillektomie war eine Literaturrecherche nach Reviews zum Thema Tonsillektomie der Jahre 2010-2014, zur Bewertung der Tonsillotomie ein Review von Windfuhr et al. aus dem Jahr 2014 [12]. Diese Arbeit wiederum inkludierte Ergebnisse aus Publikationen von 1961-2013 zum Thema Tonsillotomie nach einer systematischen Literaturrecherche. Eine Metaanalyse beinhaltet diese Arbeit von Windfuhr et al. nicht.

Die wesentlichen Aussagen und Empfehlungen der Leitlinie in Bezug auf die Tonsillenchirurgie sind folgende:

- Die Evidenzlage, dass eine Tonsillektomie als Therapie zur Besserung wiederkehrender Tonsillitis-Episoden effektiv ist, sei bei Kindern mäßig und bei Erwachsenen gering.

- Die Aussagen zur Wirksamkeit einer Tonsillektomie zur Verringerung der Häufigkeit von HalsschmerzEpisoden pro Jahr seien auf einen Nachbeobachtungszeitraum von 12 Monaten nach der Operation bei Kindern und auf 5-6 Monate bei Erwachsenen begrenzt.

- Der Einfluss einer Tonsillektomie auf die Häufigkeit der Halsschmerzen pro Jahr bei Kindern sei gering.

- Aufgrund der Heterogenität der Daten können aktuell keine eindeutigen Schlussfolgerungen zur Wirksamkeit von Tonsillektomien bei Erwachsenen gezogen werden.

- Die Auswirkungen einer Tonsillektomie auf die Lebensqualität werden als positiv eingestuft. Es seien jedoch weitere Untersuchungen erforderlich, um geeignete Bestandsaufnahmen und standardisierte Bewertungsverfahren, insbesondere bei Kindern, zu erstellen.

- Es wird postuliert, dass die Tonsillotomie im Gegensatz zur Tonsillektomie durch eine geringere postoperative Morbidität in Bezug auf Schmerzen und Blutungen gekennzeichnet sei. Obwohl Tonsillengewebe entlang der Kapsel verbleibt, wird angenommen, dass sich das Ergebnis zumindest bei Kindern und jungen Erwachsenen nicht von einer Tonsillektomie unterscheide. Diese Postulierungen unterscheiden sich deutlich von den Schlussfolgerungen des IQWiG-Berichts (siehe unten).

- Alter und Tonsillitis in der Anamnese seien keine Kontraindikationen für eine Tonsillotomie. Eine Abszessbildung in den Tonsillenresten sei ein äußerst seltener Befund.

- Die Anzahl der Episoden, die 12 Monate vor der Erstvorstellung des Patienten aufgetreten sind, sei ent- scheidend, um eine Tonsillektomie oder Tonsillotomie als therapeutische Option anzuzeigen.

- Während eine Operation bei Patienten mit weniger als 3 Episoden nicht angezeigt sei, sei eine Wartezeit von 6 Monaten gerechtfertigt, um das Potenzial einer spontanen Heilung zu berücksichtigen, bevor eine Operation in Betracht gezogen werden sollte. Mindestens 6 Episoden würden auf eine Tonsillektomie (oder ggf. auch eine Tonsillotomie) als therapeutische Option hindeuten.

Die Gültigkeit der Leitlinie läuft ab, und die Überarbeitung ist derzeit in Vorbereitung. Momentan werden in der Leitlinie Tonsillotomie und Tonsillektomie als alternative Verfahren zur Behandlung der rezidivierenden akuten Tonsillitis, wenn die genannten Kriterien erfüllt sind, gleichgesetzt. Mittlerweile gibt es den IQWiG-Bericht, dessen Bewertung der Literatur methodisch über die Leitlinie hinausgeht. Damit wird sich die überarbeitete Leitlinie sicher auseinandersetzen und differenziertere Empfehlungen für das eine oder andere Verfahren abgeben müssen.

Merke

Die Veröffentlichung der S2k-Leitlinie „Therapie entzündlicher Erkrankungen der Gaumenmandeln - Tonsillitis“ war ein wichtiger Schritt, die Diskussion auf ein medizinisch-wissenschaftliches Niveau zu führen, die Wissenslücken zu beschreiben und Studien auf den Weg zu bringen.

\section{IQWiG-Bericht zur Tonsillotomie bei rezidivie- render akuter Tonsillitis und bei Hyperplasie der Tonsillen}

Das IQWiG hat im März 2017 den Abschlussbericht zur „Tonsillotomie bei rezidivierender akuter Tonsillitis und bei Tonsillenhyperplasie“ vorgelegt (Projekt N15-11). Der Auftraggeber war der G-BA mit mehreren Zielen, nämlich einer Nutzenbewertung der Tonsillotomie im Vergleich zur konservativen Behandlung (z. B. Watchful Waiting) und der Nutzenbewertung einer Tonsillotomie im Vergleich zur Tonsillektomie bei 2 Krankheitsbildern:

- Patienten mit rezidivierender akuter Tonsillitis und

- Patienten mit Hyperplasie der Tonsillen.

Als patientenrelevante Endpunkte wurden definiert: Mortalität, Morbidität, insbesondere Blutungen, Schmerz, rezidivierende Tonsillitis und HNO-Infektionen, Schluckund Schlafstörungen, Krankenhausaufenthaltsdauer/ erneute Hospitalisierung, erneute Tonsillenoperation, gesundheitsbezogene Lebensqualität sowie sonstige unerwünschte Wirkungen der Therapie. Basierend auf einer systematischen Literaturrecherche wurden ausschließlich randomisierte kontrollierte Studien bis einschließlich November 2016 analysiert. 
Die Schlussfolgerungen des IQWiG weichen teilweise von den Empfehlungen der AWMF-S2k-Leitlinie ab. Die wichtigsten Schlussfolgerungen des Berichts sind:

- Zum Vergleich Tonsillotomie versus konservative Behandlung ließen sich weder relevante noch laufende Studien identifizieren, also war keine Aussage zu treffen.

- Generell habe die Tonsillotomie im Vergleich zur Tonsillektomie kurzfristige Vorteile für 2 Wochen: Es gebe Hinweise und gelegentlich geringe Anzeichen dafür, dass Schmerzen, Schluckstörungen und Schlafstörungen nach Tonsillotomie im Vergleich zur Tonsillektomie seltener auftreten.

- Für die anderen patientenrelevanten Endpunkte wie postoperative Blutungen, Krankenhausaufenthaltsdauer, Hospitalisierung, gesundheitsbezogene Lebensqualität und sonstige unerwünschte Wirkungen habe sich kein Anhaltspunkt für einen höheren oder geringeren Nutzen oder Schaden zwischen den Behandlungen ergeben. Für den Endpunkt Mortalität seien keine Daten verfügbar gewesen.

- Bei der Indikation rezidivierende akute Tonsillitis habe sich ein Anhaltspunkt für einen geringeren Nutzen der Tonsillotomie gegenüber der Tonsillektomie ergeben.

- Bei der Indikation rezidivierende akute Tonsillitis und der Indikation Tonsillenhyperplasie würden Langzeitdaten fehlen.

- Die Datenlage für den Endpunkt erneute Tonsillenoperation sei unzureichend gewesen.

Die Bewertung des Berichts durch den G-BA führte zu dem Richtlinien-Beschluss, die Tonsillotomie als Regelleistung zur Behandlung der krankhaften Hyperplasie der Gaumenmandeln ab dem vollendeten 1. Lebensjahr einzuführen, wenn die Hyperplasie eine symptomatische, klinisch relevante Beeinträchtigung bewirke und eine konservative Behandlung nicht ausreiche. Dagegen schlussfolgerte der G-BA, dass die Datenlage keine Nutzenbewertung der Tonsillotomie zur Behandlung der rezidivierenden akuten Tonsillitis erlaube.

\section{FAZIT}

Der IQWiG-Bericht zur Tonsillotomie macht deutlich, wie wenig evidenzbasierte hochwertige Studien zum Thema vorliegen, obwohl die Tonsillenchirurgie weltweit ein häufiger Eingriff ist. Dem HNO-Arzt werden andererseits auch die methodischen Grenzen der evidenzbasierten Methodik vor Augen geführt, wenn man die Tonsillotomie und Tonsillektomie ausschließlich anhand von randomisierten kontrollierten Studien bewertet; folgt man konsequent der Methodik und Logik des IQWiG, so sind noch nicht einmal das verminderte Blutungsrisiko und die geringere Schwere der postoperativen Schmerzen für die Tonsillotomie belegt.

\section{G-BA-Erprobungsstudie zur Tonsillotomie und weitere laufende Studien}

Im Dezember 2018 erfolgte durch den G-BA der Richtlinienbeschluss, dass in einer klinischen Studie die Erprobung der Tonsillotomie zur Behandlung der rezidivierenden akuten Tonsillitis notwendig sei, mit dem Ziel beantworten zu können, ob eine Tonsillotomie einer Tonsillektomie nicht unterlegen sei. Als Ergebnis eines Ausschreibungsverfahrens wurde schließlich im Dezember 2019 eine Bietergemeinschaft aus Universitätsklinikum Jena, Deutscher Gesellschaft für Hals-Nasen-Ohren-Heilkunde, Kopf- und Hals-Chirurgie e. V. und dem Deutschen Berufsverband der Hals-Nasen-Ohrenärzte e. V. vom G-BA beauftragt, die geplante Studie zur Tonsillotomie wissenschaftlich zu begleiten und die Ergebnisse auszuwerten. Derzeit wird das Studienprotokoll erarbeitet und die Einreichung vor einer Ethikkommission vorbereitet. Die Studie wird lauten: „Tonsillektomie versus Tonsillotomie bei Kindern und Erwachsenen mit rezidivierender akuter Tonsillitis (Akronym: TOTO): Eine kontrollierte, randomisierte Nichtunterlegenheitsstudie“.

Auf www.ClinicalTrial.gov fanden sich am 21.03.2020 unter dem Stichwort „tonsillotomy“ 7 Studien. Darunter fanden sich 2 Studien, die eine Tonsillotomie mit einer Tonsillektomie bei Kindern mit obstruktivem SchlafApnoe-Syndrom (OSAS) verglichen. Unter dem Stichwort „tonsillectomy“ waren 235 Studien registriert. Darunter fanden sich vor allem Studien zu verschiedenen instrumentellen Techniken, also Medizinprodukte-Studien und solche zu Schmerzen nach der Operation.

Eine wichtige, derzeit rekrutierende, multizentrische Studie ist der „NAtional randomised controlled Trial of Tonsillectomy IN Adults“ (Akronym: NATTINA) [13]. Die NATTINA-Studie vergleicht eine konservative Therapie mit abwartender Haltung gegenüber einer direkten Tonsillektomie bei Erwachsenen mit rezidivierender akuter Tonsillitis und hat ein 24-Monate-Follow-up vorgesehen. Zwischenauswertungen sind bislang nicht publiziert worden. Die wesentlichen Parameter der NATTINA-Studie und der TOTO-Studie sind in $>$ Tab. 2 gegenübergestellt.

Die Schwedische HNO-Gesellschaft hat bereits 1997 ein nationales Register für alle Tonsilleneingriffe für gutartige Erkrankungen gestartet und sammelt umfangreiche Daten insbesondere seit 2009 (National Tonsil Surgery Registry in Sweden, NTSRS: https://ton.registercentrum. se). Das NTSRS geht davon aus, etwa $80 \%$ der Eingriffe zu umfassen. Das Register versucht Follow-up-Daten bis 6 Monate zu erfassen und hatte 2018120000 Patienten inkludiert [14]. Die Struktur wird derzeit als Vorbild für den Aufbau eines gemeinsamen skandinavischen Registers genutzt (Nordic Tonsil Surgery Register Collaboration) [14]. Das NTSRS konnte z. B. aus den Daten von 2004-2013 zeigen, dass die Inzidenz für eine erneute 
- Tab. 2 Gegenüberstellung von NATTINA (NAtional randomised controlled Trial of Tonsillectomy IN Adults) [13] und TOTO (Tonsillektomie versus Tonsillotomie bei Kindern und Erwachsenen mit rezidivierender akuter Tonsillitis).

\begin{tabular}{|c|c|c|}
\hline & NATTINA & тото \\
\hline Sponsor & National Health Service (NHS), UK & Gemeinsamer Bundesausschuss (G-BA) \\
\hline Studienleitung & Newcastle Clinical Trials Unit, Newcastle University & $\begin{array}{l}\text { Deutsche Gesellschaft für Hals-Nasen-Ohren-Heilkunde, } \\
\text { Kopf- und Hals-Chirurgie e. V., Deutscher Berufsverband } \\
\text { der Hals-Nasen-Ohrenärzte e. V., HNO-Klinik des Univer- } \\
\text { sitätsklinikums Jena }\end{array}$ \\
\hline Internetseite & https://research.ncl.ac.uk/nattina/ & in Vorbereitung \\
\hline Studienziel & $\begin{array}{l}\text { Vergleich der klinischen und gesundheitsökonomi- } \\
\text { schen Effektivität der Tonsillektomie mit einer } \\
\text { konservativen Therapie }\end{array}$ & $\begin{array}{l}\text { Bestimmung der Nichtunterlegenheit der Tonsillotomie } \\
\text { im Vergleich zu der Tonsillektomie bei der Behandlung } \\
\text { der rezidivierenden akuten Tonsillitis }\end{array}$ \\
\hline Studiendesign & $\begin{array}{l}\text { randomisierte multizentrische, 2-armige, unverblindete } \\
\text { Studie }\end{array}$ & $\begin{array}{l}\text { randomisierte multizentrische, 2-armige, unverblindete } \\
\text { Nichtunterlegenheitsstudie }\end{array}$ \\
\hline Intervention & Tonsillektomie & Tonsillotomie \\
\hline Kontrolle & konservative Therapie & Tonsillektomie \\
\hline Haupteinschlusskriterium & $\begin{array}{l}\text { Alter: } \geq 16 \text { Jahre; rezidivierende akute Tonsillitis nach } \\
\text { den Kriterien der SIGN-Leitlinie Tonsillitis [32] }\end{array}$ & $\begin{array}{l}\text { Alter: } \geq 3 \text { Jahre; rezidivierende akute Tonsillitis nach den } \\
\text { Kriterien der AWMF-Leitlinie Tonsillitis }\end{array}$ \\
\hline Follow-up & 24 Monate & 24 Monate \\
\hline primärer Endpunkt & $\begin{array}{l}\text { Anzahl symptomatischer Entzündungen im Hals-Rachen- } \\
\text { Raum innerhalb von } 24 \text { Monaten }\end{array}$ & $\begin{array}{l}\text { Anzahl symptomatischer Entzündungen im Hals-Rachen- } \\
\text { Raum innerhalb von } 24 \text { Monaten nach Operation }\end{array}$ \\
\hline Fallzahl & Screening: 528 Patienten & Screening: 568 Patienten; ITT: 454; PP: 362 \\
\hline Prüfzentren & geplant 9, am Ende 22 & geplant 20 \\
\hline Studienstand & last patient in: 30.04 .2018 & geplant 2. Jahreshälfte 2020 \\
\hline
\end{tabular}

Operation nach Tonsillotomie bei 1,1 pro 1000 Personen pro Jahr lag [15]. Unter den 35060 von 2007-2015 registrierten Patienten lag die Nachblutungsrate binnen 30 Tagen mit Blutstillung im Operationssaal nach Tonsillotomie bei 0,1\% und nach Tonsillektomie bei 0,5\% [16].

Die Daten sind wertvoll, weil vor allem wissenschaftliche Aussagen über die Versorgungslandschaft getroffen werden können. Wie bei allen Registerdaten sind kausale Aussagen nur begrenzt möglich. Eine Stärke ist ganz klar die Erfassung von Daten zur Tonsillotomie. In Deutschland werden über das Statistische Bundesamt und die DRG-Daten qualitätsgesichert nur die stationären Patienten erfasst ( $\triangleright$ Abb. $\mathbf{3}$ ), sodass gute Aussagen zur Tonsillektomie, aber nicht zur Tonsillotomie möglich sind, die häufig ambulant erbracht wird [7].

Populationsbezogene Daten über DRG-Analysen hinaus liegen ebenso nur für den stationären Bereich vor: In Thüringen lag 2012 nach stationären Eingriffen die Reoperationsrate nach Tonsillotomie bei 0,2\% [17]. Zur Analyse der Tonsillotomie sind aufwendigere Sekundärdatenanalysen aus Krankenkassendaten notwendig, mit begrenzter Möglichkeit wissenschaftlicher Auswertung [18].

\section{Merke}

Betrachtet man die laufenden prospektiven, randomisiert kontrollierten Studien, so wird es noch 2-3 Jahre dauern, bis wir in Bezug auf die Rolle der Chirurgie zur Behandlung der rezidivierenden akuten Tonsillitis einen relevanten neuen Kenntnisstand haben werden.

\section{G-BA-Richtlinie zum Zweitmeinungsverfahren}

Im Dezember 2017 trat die Richtlinie des G-BA zum Zweitmeinungsverfahren in Kraft (www.g-ba.de/beschlu esse/3079/). Neben der Hysterektomie galt ab sofort für die Tonsillektomie und die Tonsillotomie für die Patienten oder Erziehungsberechtigten ein Anspruch auf Einholung einer unabhängigen ärztlichen Zweitmeinung (2019 ist die Schulterarthroskopie hinzugekommen).

Es handelt sich um ein freiwilliges Verfahren. Der HNOArzt, der eine Tonsillektomie oder Tonsillotomie für indiziert hält, muss den Hinweis oder ggf. Verzicht des Patienten auf das Zweitmeinungsverfahren dokumentieren. Die Zweitmeinung kann nur bei einem von der zuständigen kassenärztlichen Vereinigung zugelassenen Zweitmeiner eingeholt werden. Der Zweitmeiner darf später nicht die Operation vornehmen, sollte er diese denn auch empfehlen. 


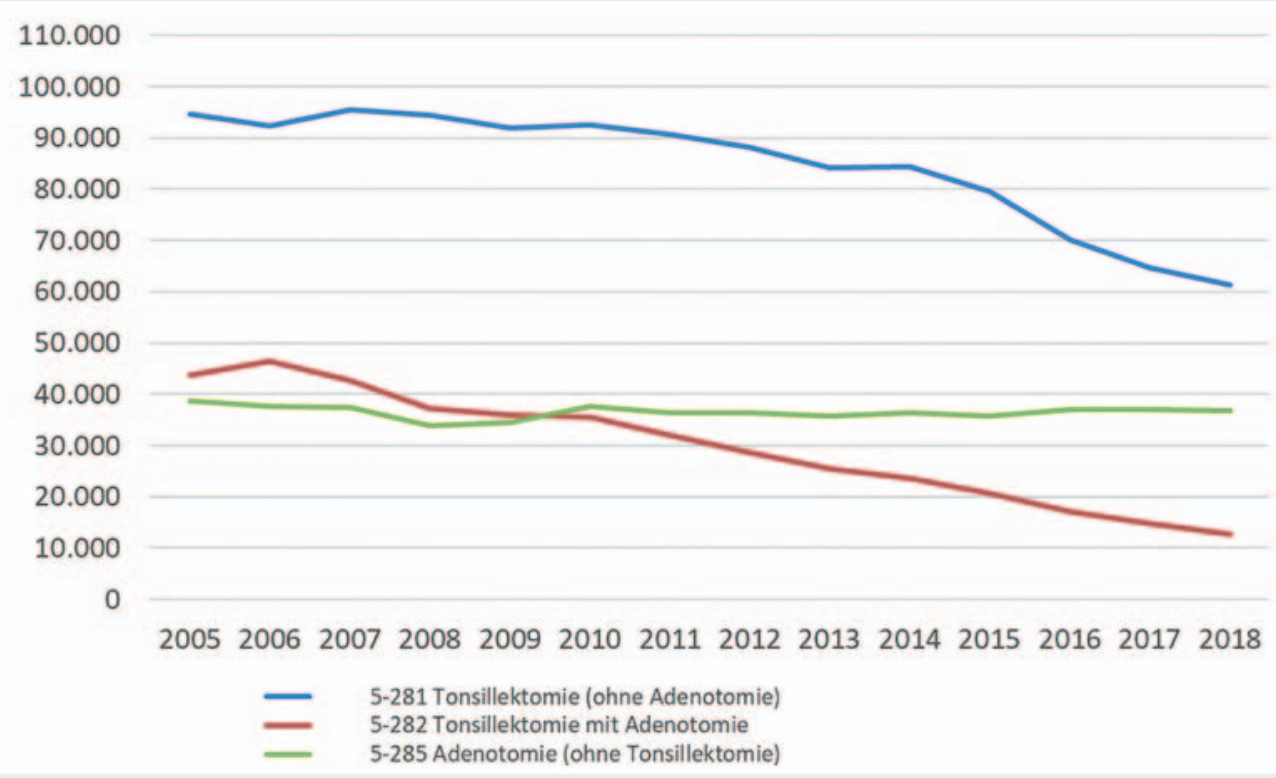

- Abb. 3 Entwicklung der stationären Fallzahlen für Tonsillektomie und Adenotomie in Deutschland von 2005-2018. Aus: Gesundheitsberichterstattung des Bundes, Operationen und Prozeduren der vollstationären Patientinnen und Patienten in Krankenhäusern (www.gbe-bund.de). Während die Anzahl der Tonsillektomien bis heute kontinuierlich abnimmt, bleibt die Rate an Adenotomien konstant. Wichtig: Ambulante Operationen sind nicht erfasst, sodass hierüber keine gute Statistik über die Tonsillotomien erfolgen kann.

Das IQWiG unterhält die Internetseite www.gesundheits information.de zur öffentlichen Gesundheitsaufklärung. Dort finden sich Aufklärungsmaterial zur Tonsillitis und zur Tonsillenhyperplasie sowie Entscheidungshilfen mit Gegenüberstellung der Operationsverfahren mit ihren Vor- und Nachteilen.

\section{FAZIT}

Das Zweitmeinungsverfahren geht auf das Gesetz zur Stärkung der Versorgung in der gesetzlichen Krankenversicherung (Versorgungsstärkungsgesetz) zurück. Primär zielt das Gesetz dabei auf mengenanfällige planbare Eingriffe. Wie häufig eine Zweitmeinung eingeholt wird, und wie häufig die Zweitmeinung das Verhalten der Patienten für oder gegen einen Eingriff an den Tonsillen verändert, ist unbekannt.

\section{Aufnahme der Tonsillotomie in den AOP-Katalog}

Der G-BA hatte 2018, wie bereits beschrieben, die Tonsillotomie als Leistung der gesetzlichen Krankenkassen für die Behandlung einer symptomatischen und klinisch relevanten Tonsillenhyperplasie im Krankenhaus und in der ambulanten Medizin zugelassen. Im ambulanten Sektor werden Anforderungen für Operationen über den Katalog ambulant durchführbarer Operationen (AOP-Katalog) geregelt.
Im Moment steht noch die Festlegung aus, ob die Tonsillotomie als obligat ambulant (Kategorie 1) oder optional ambulant (Kategorie 2) eingeordnet wird. Die Deutsche Gesellschaft für Hals-Nasen-Ohren-Heilkunde, Kopf- und Hals-Chirurgie e. V. vertritt nach ausführlicher Bewertung die Ansicht, dass die Tonsillotomie nur in Kategorie 2 eingeordnet werden könne. Medizinische Gründe, HNO-chirurgische Aspekte, anästhesiologische Aspekte und Aspekte der postoperativen Betreuung würden eine Einordnung in Kategorie 1 verbieten, genauso wie ökonomische Gründe [19]. Die Entscheidung des Gesetzgebers gilt es abzuwarten.

\section{Offene Fragen}

Die wichtigsten offenen Fragen sind in $>$ Tab. 3 zusammengefasst. Im Einzelnen sollte die weitere Forschung zur Tonsillenchirurgie die nachfolgenden Themen bearbeiten.

\section{Tonsillotomie versus Tonsillektomie versus konservative Therapie}

Auch wenn die Tonsillektomie und Tonsillotomie als über viele Jahre bewährte chirurgische Verfahren in der HNOHeilkunde anzusehen sind, die tagtäglich weltweit häufig eingesetzt werden, sind viele Fragen zur Indikation offen. Der direkte Vergleich der Verfahren zum Einsatz bei der rezidivierenden akuten Tonsillitis wird demnächst durch die G-BA-Erprobungsstudie erfolgen. Die NATTINA-Studie 
- Tab. 3 Offene Fragen rund um die Tonsillenchirurgie.

\begin{tabular}{|c|c|c|}
\hline Thema & Frage & Kommentar \\
\hline \multirow[t]{2}{*}{ Operationstechnik } & Tonsillotomie oder Tonsillektomie? & $\begin{array}{l}\text { Tonsillenhyperplasie: Nutzen-Risiko-Ratio spricht für Tonsillotomie, } \\
\text { wobei die Evidenz nicht hoch ist. } \\
\text { Rezidivierende akute Tonsillitis: Nutzen-Risiko-Ratio im Vergleich } \\
\text { nicht geklärt. }\end{array}$ \\
\hline & Welches Schneideverfahren? & $\begin{array}{l}\text { Bislang gibt es keinen Hinweis, dass ein besonderes Verfahren einem } \\
\text { anderen überlegen ist (Kaltinstrumente, Laser, Radiofrequenz etc.). }\end{array}$ \\
\hline Definition & Was versteht man unter Tonsillotomie? & $\begin{array}{l}\text { Wie viel Tonsille bei einer Tonsillotomie entfernt werden sollte, ist } \\
\text { nicht geklärt. }\end{array}$ \\
\hline Indikation & Was sind Kriterien für eine Operation? & $\begin{array}{l}\text { Valide Parameter zur Indikationsstellung, z. B. einen Biomarker, gibt } \\
\text { es bislang nicht. Solange sind die Kriterien, wie in der Leitlinie } \\
\text { aufgegriffen, das Maß der Dinge. }\end{array}$ \\
\hline perioperative Behandlung & $\begin{array}{l}\text { Für welche Patienten ist eine perioperative } \\
\text { Antibiose sinnvoll? }\end{array}$ & Hier ist die Datenlage sehr widersprüchlich. \\
\hline postoperative Behandlung & $\begin{array}{l}\text { Wie sieht das optimale Schmerzmanage- } \\
\text { ment aus? }\end{array}$ & $\begin{array}{l}\text { Viele Patienten haben immer noch zu starke postoperative Schmerzen } \\
\text { nach Tonsillektomie. }\end{array}$ \\
\hline \multirow[t]{3}{*}{ Qualitätsmanagement } & Wie könnten gute Qualitätsziele aussehen? & $\begin{array}{l}\text { Qualitätsziele jenseits von Operationskomplikationen und Schmerz } \\
\text { sollten definiert werden. }\end{array}$ \\
\hline & Was bringt das Zweitmeinungsverfahren? & $\begin{array}{l}\text { Dies muss unbedingt geprüft und bei mangelnder Effektivität wieder } \\
\text { abgeschafft werden. }\end{array}$ \\
\hline & $\begin{array}{l}\text { Wie kann die regionale Variabilität der } \\
\text { Häufigkeit der Tonsillenchirurgie gesenkt } \\
\text { werden? }\end{array}$ & $\begin{array}{l}\text { Mit der neuen Leitlinie, den nächsten Studienergebnissen und der } \\
\text { Definition von Qualitätszielen wird dieses Thema an Relevanz verlieren. }\end{array}$ \\
\hline
\end{tabular}

wird neue Erkenntnisse bringen zum Vergleich der Tonsillenchirurgie mit konservativer Therapie.

\section{Studien zur Tonsillenchirurgie bei kindlichem OSAS}

Auch wenn die Tonsillotomie nunmehr als Regelleistung zur Behandlung der symptomatischen Tonsillenhyperplasie zugelassen ist, sollten wir uns vergegenwärtigen, dass die meisten Studien zur Behandlung des OSAS mit Tonsillenchirurgie die Tonsillektomie als Standardverfahren verwenden [20]. Wenn auch aktuelle Daten nahelegen, dass die Tonsillotomie hier zumindest gleichwertig ist, fehlen große vergleichende klinische Studien [21].

\section{Bessere Indikationskriterien für eine Tonsillen- chirurgie bei rezidivierender akuter Tonsillitis}

Unabhängig von der Wahl des operativen Verfahrens, sei es Tonsillotomie oder Tonsillektomie, benötigen wir bessere Parameter für die Indikationsstellung bei Patienten mit rezidivierender akuter Tonsillitis. Ein einfaches Zählverfahren von Episoden pro Zeiteinheit, wie derzeit durch die Leitlinie empfohlen, kann nur unzuverlässig sein, insbesondere wenn auch die Antibiotikapflichtigkeit mit einbezogen wird. Dies geht nämlich von der Annahme aus, daran die Schwere der Erkrankung messen zu können, wofür es keinen Anhalt gibt. Zudem wird die Verordnung von Antibiotika bei einer Tonsillitis sehr variabel gehand- habt. Letztendlich ist unklar, warum manche Personen gehäuft eine Tonsillitis erleiden und andere nicht. Der Idealfall wäre z. B. ein Biomarker im Blut. Leider gibt es bislang keinen verlässlichen Blutmarker [22].

\section{Besseres postoperatives Schmerzmanagement}

Ein weiteres relevantes Problem ist das postoperative Schmerzmanagement nach Tonsillektomie. Die Tonsillektomie gehört zu den schmerzhaftesten operativen Eingriffen in der HNO-Heilkunde [23]. Bislang gibt es kein zufriedenstellendes Regime, und eine Lösung ist bislang nicht in Sicht [24].

\section{Definition von Qualitätszielen der Tonsillenchirurgie}

Der G-BA hat sich bereits vor der Publikation des Faktenchecks Gesundheit der Bertelsmann Stiftung mit dem Thema eines sektorenübergreifenden Qualitätssicherungsverfahrens beschäftigt und 2013 das Institut für angewandte Qualitätsförderung und Forschung im Gesundheitswesen (AQUA) beauftragt, eine Konzeptskizze zu erstellen. Das AQUA-Institut empfiehlt als Qualitätsziele: Indikationsstellung, Komplikationen wie postoperative Blutungen, Schmerzen, postoperative Nausea and Vomiting (PONV), prä- und postoperative Aufklärung bzw. gemeinsame Entscheidungsfindung, perioperative Antibiotikaprophylaxe und Notfallmanagement. 
- Tab.4 Mögliche Instrumente für die Entwicklung von Qualitätskriterien: Fragebögen zur Lebensqualität und Patient-reported Outcome Measures (PROM).

\begin{tabular}{|c|c|c|c|}
\hline Fragebogen & Zielgruppe & $\begin{array}{l}\text { Validierung in } \\
\text { deutscher Sprache }\end{array}$ & Kommentar \\
\hline \multicolumn{4}{|l|}{ allgemeine Lebensqualität } \\
\hline SF-12/SF-36 [33-35] & Erwachsene & ja & $\begin{array}{l}\text { ermöglicht den Vergleich mit Normal- } \\
\text { personen und Patienten mit anderen } \\
\text { Erkrankungen }\end{array}$ \\
\hline KINDL-R/KIDSCREEN-10 [36, 37] & Kinder & ja & $\begin{array}{l}\text { ermöglicht den Vergleich mit Normal- } \\
\text { personen und Patienten mit anderen } \\
\text { Erkrankungen }\end{array}$ \\
\hline \multicolumn{4}{|l|}{ allgemeine Operationsfragebögen } \\
\hline Glasgow Benefit Inventory (GBI) [38, 39] & Erwachsene & ja & $\begin{array}{l}\text { Ex-post-Bewertung der Verbesserung } \\
\text { durch die Operation }\end{array}$ \\
\hline $\begin{array}{l}\text { Glasgow Children's Benefit Inventory } \\
\text { (GCBI) }[40,41]\end{array}$ & Eltern der Kinder & ja & $\begin{array}{l}\text { Ex-post-Bewertung der Verbesserung } \\
\text { durch die Operation }\end{array}$ \\
\hline \multicolumn{4}{|c|}{ krankheitsspezifische/therapiespezifische Fragebögen } \\
\hline Obstructive sleep apnea-18 (OSA-18) [42] & Eltern der Kinder & ja & für Kinder mit OSAS \\
\hline Obstructive sleep disorders-6 OSD-6 [43] & Eltern der Kinder & nein & für Kinder mit OSAS \\
\hline $\begin{array}{l}\text { Specific Benefits from Tonsillectomy } \\
\text { Inventory SBTI [44] }\end{array}$ & Eltern der Kinder & ja & $\begin{array}{l}\text { Ex-post-Bewertung der Verbesserung } \\
\text { durch die Tonsillenoperation }\end{array}$ \\
\hline $\begin{array}{l}\text { Tonsil and Adenoid Health Status } \\
\text { Instrument TAHSI }[45,46]\end{array}$ & $\begin{array}{l}\text { Original: Kinder 2-16 Jahre; } \\
\text { deutsch: Erwachsene }\end{array}$ & $\begin{array}{l}\text { Kinder: nein; } \\
\text { Erwachsene: ja }\end{array}$ & - \\
\hline $\begin{array}{l}\text { Tonsillectomy Outcome Inventory } 14 \\
\text { TOI-14 [47] }\end{array}$ & Erwachsene & ja & für die Tonsillektomie validiert \\
\hline \multicolumn{4}{|l|}{ postoperativer Schmerz } \\
\hline $\begin{array}{l}\text { Qualitätsverbesserung in der postopera- } \\
\text { tiven Schmerztherapie (QUIPS) [48] }\end{array}$ & Erwachsene & ja & $\begin{array}{l}\text { neben Rating-Skalen auch Erfassung se- } \\
\text { kundärer Schmerz-assoziierter Parameter }\end{array}$ \\
\hline $\begin{array}{l}\text { Qualitätsverbesserung in der postopera- } \\
\text { tiven Schmerztherapie von Kindern } \\
\text { QUIPSI [49] }\end{array}$ & Kinder $\geq 4$ Jahre & ja & $\begin{array}{l}\text { neben Rating-Skalen auch Erfassung se- } \\
\text { kundärer Schmerz-assoziierter Parameter }\end{array}$ \\
\hline
\end{tabular}

Es wurde damals aufgrund mangelnder Evidenz festgelegt, zunächst die Leitlinie abzuwarten und danach weitere Empfehlungen auszusprechen [25]. Nun steht bereits die Überarbeitung der Leitlinie an, und es wäre wünschenswert, wenn die HNO-Heilkunde aus sich heraus Qualitätsziele für die Tonsillenchirurgie definieren würde. Wichtig wäre auch der standardisierte Einsatz von Fragebögen zur Lebensqualität und anderer Patient-reported Outcome Measures (PROM; Übersicht in [26]; > Tab.4).

\section{Reduktion der Variabilität? Oder bessere personalisierte Entscheidungsfindung?}

Auch nach nun längerer Einführung der Leitlinie beobachten wir immer noch eine erhebliche geografische Variabilität bei den Operationsraten in Deutschland [7]. Einen akzeptablen Grad der Variabilität zu definieren ist schwierig [27]. Vielmehr steht im Vordergrund, basierend auf möglichst vielen Informationen und Parametern, für den individuellen Patienten eine qualitätsgesicherte personalisierte Entscheidung zu treffen [28]. Was uns neben Bio- markern hierfür fehlt, ist das Wissen um die Präferenzen der Patienten.

Vor kurzem wurden 234 Eltern befragt, ob sie sich für eine Tonsillotomie oder eine Tonsillektomie entscheiden würden, wenn die Gaumenmandeln operiert werden müssten. Vorgelegt wurde eine Auflistung von Argumenten für und gegen den jeweiligen Eingriff. 86 \% bevorzugen hypothetisch die Tonsillektomie, weil das Risiko der Möglichkeit der erneuten Operation sehr bedeutsam war [29]. Bemängelt wurde an der Studie die Qualität der Information, die suggestiv per se eher zur Entscheidung für eine Tonsillektomie geführt hätte [30]. Zu den Präferenzen bzw. Erwartungen an die Operation von den Eltern und Patienten in Deutschland ist gar nichts bekannt.

Langsam bekommen wir ein klareres Bild von der Rolle der Tonsillotomie in Abgrenzung zur Tonsillektomie. Als nächstes bedeutsam ist sicherlich die anstehende Überarbeitung der Leitlinie. Unterstützung durch Software als 
sog. Clinical Decision Support System (CDSS), welche die individuellen Daten, die Präferenz des Patienten und die Empfehlungen der Leitlinie zusammenbringt, wäre für den HNO-Arzt zur Indikationsstellung einer Tonsillenchirurgie in Zukunft wichtig [31].

\section{KERNAUSSAGEN}

- Die Tonsillektomie ist ein genau definierter Eingriff. Die Tonsillotomie ist nicht klar definiert, wenn auch zumeist das Gewebe medial des $\mathrm{Ni}$ veaus der Gaumenbögen entfernt wird.

- Die Tonsillotomie hat einen gut belegten Nutzen bei der symptomatischen Hyperplasie der Gaumenmandeln bei Kindern. Der Nutzen scheint der Tonsillektomie gleichwertig, die Risiken sind dagegen geringer.

- Der Nutzen der Tonsillotomie zur Behandlung der rezidivierenden akuten Tonsillitis ist unklar.

- Forschung zum Nutzen oder möglicherweise mangelnden Nutzen des Zweitmeinungsverfahrens ist notwendig.

- Es gilt gemeinhin für die Tonsillenchirurgie, Qualitätsziele zu definieren und die Präferenz der Patienten/Eltern in die Entscheidungsfindung einzubeziehen.

\section{Wissenschaftlich verantwortlich gemäß Zertifizierungsbestimmungen}

Wissenschaftlich verantwortlich gemäß Zertifizierungsbestimmungen für diesen Beitrag ist Prof. Dr. med. Orlando Guntinas-Lichius, Jena.

\section{Interessenkonflikt}

Die Autorinnen/Autoren geben an, dass kein Interessenkonflikt besteht.

\section{Autorinnen/Autoren}

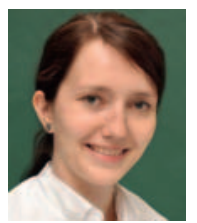

\section{Katharina Geißler}

Dr. med., 2004-2010 Medizinstudium und 2012 Promotion an der Martin-Luther-Universität Halle-Wittenberg. 2011-2016 Weiterbildung zur Fachärztin für Hals-, Nasen- und Ohrenheilkunde, seit 2017 Oberärztin an der Klinik und Poliklinik für Hals-Nasen-Ohrenheilkunde des Universitätsklinikum Jena. Klinische Schwerpunkte: effektive Schmerzbehandlung nach Tonsillektomie, Immunologie, Allergologie, Pädiatrische HNO-Heilkunde.

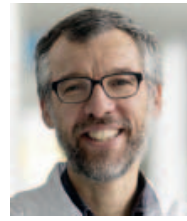

\section{Orlando Guntinas-Lichius}

Univ.-Prof. Dr. med., 1987-1993 Studium der Humanmedizin in Köln, 1994 Promotion. Ab 1994 Arzt im Praktikum bzw. Assistenzarzt in der Hals-Nasen-Ohrenklinik der Universität zu Köln. 1998 Anerkennung der Gebietsbezeichnung HNO-Heilkunde. 2001 Habilitation für das Fach HNO-Heilkunde und Ernennung zum Oberarzt. 2003 Leitender Oberarzt. 2006 Apl. Prof. der Universität zu Köln 2006. Seit 2006 W3-Professur für HNO-Heilkunde an der Universität Jena und Direktor der dortigen HNO-Klinik. Wissenschaftliche Schwerpunkte: neue diagnostische Verfahren und klinische Prüfungen zur multimodalen Therapie von Kopf-Hals-Tumoren, neue Verfahren zur Behandlung der Fazialisparese, HNO-Versorgungsforschung, postoperativer Schmerz.

\section{Korrespondenzadresse}

Univ.-Prof. Dr. med. Orlando Guntinas-Lichius

Klinik und Poliklinik für Hals-Nasen-Ohrenheilkunde Universitätsklinikum Jena

Am Klinikum 1

D-07747 Jena

orlando.guntinas@med.uni-jena.de

\section{Literatur}

[1] Nolting HD, Zich K, Deckenbach B. Faktencheck Gesundheit. Entfernung der Gaumenmandeln bei Kindern und Jugendlichen. Gütersloh: Bertelsmann Stiftung. 2013

[2] AWMF-Leitlinie: Therapie entzündlicher Erkrankungen der Gaumenmandeln - Tonsillitis. 2016. Nr. 017/024

[3] Geissler K, Guntinas-Lichius O. Surgery in the Pharynx: Adenoidectomy, Tonsillectomy, Tonsillotomy and Surgery for Obstructive Sleep Apnea. Laryngorhinootologie 2016; 95 : 132-143

[4] Feldmann H. 2000 year history of tonsillectomy. Images from the history of otorhinolaryngology, highlighted by instruments from the collection of the German Medical History Museum in Ingolstadt. Laryngorhinootologie 1997; 76: 751 760

[5] Eisfeld W, Deitmer T. From tonsil capping to tonsillectomy to tonsillotomy. Laryngorhinootologie 2010; 89: 137-144

[6] Scherer $\mathrm{H}$. Tonsillotomy versus tonsillectomy. Laryngorhinootologie 2003; 82: 754-755

[7] Windfuhr JP, Chen YS. Tonsil surgery in the federal states of Germany: similarities and differences. HNO 2020; 68: 33-42

[8] Grob GN. The rise and decline of tonsillectomy in twentiethcentury America. J Hist Med Allied Sci 2007; 62: 383-421

[9] Glover JA. The incidence of tonsillectomy in school children. 1938. Int J Epidemiol 2008; 37: 9-19

[10] Chen AY. Redirecting, Without Dampening, the Enthusiasm of Surgeons. JAMA Otolaryngol Head Neck Surg 2019. doi:10.1001/jamaoto.2019.2404

[11] Birkmeyer JD, Reames BN, McCulloch P et al. Understanding of regional variation in the use of surgery. Lancet 2013; 382: 1121-1129

[12] Windfuhr JP, Savva K, Dahm JD et al. Tonsillotomy: facts and fiction. Eur Arch Otorhinolaryngol 2014; 272: 949-969 
[13] Rubie I, Haighton C, O'Hara J et al. The NAtional randomised controlled Trial of Tonsillectomy IN Adults (NATTINA): a clinical and cost-effectiveness study: study protocol for a randomised control trial. Trials 2015; 16: 263

[14] Ruohoalho J, Ostvoll E, Bratt M et al. Systematic review of tonsil surgery quality registers and introduction of the Nordic Tonsil Surgery Register Collaboration. Eur Arch Otorhinolaryngol 2018; 275: 1353-1363

[15] Sunnergren O, Odhagen E, Stalfors J. Incidence of second surgery following pediatric adenotonsillar surgery: a populationbased cohort study. Eur Arch Otorhinolaryngol 2017; 274: 2945-2951

[16] Odhagen E, Stalfors J, Sunnergren O. Morbidity after pediatric tonsillotomy versus tonsillectomy: A population-based cohort study. Laryngoscope 2019; 129: 2619-2626

[17] Mueller J, Boeger D, Buentzel J et al. Population-based analysis of tonsil surgery and postoperative hemorrhage. Eur Arch Otorhinolaryngol 2015; 272: 3769-3777

[18] Windfuhr JP, Gerste B. Trends, regionale Variabilität und Indikationsstellung von Tonsillektomien in Deutschland. Stuttgart: Schattauer; 2015

[19] Guntinas-Lichius O, Windfuhr J, Deitmer T et al. Statement of the German Society of Otorhinolaryngology, Head and Neck Surgery on the execution of a tonsillotomy. Laryngorhinootologie 2020; 99: 176-180

[20] Venekamp RP, Hearne B], Chandrasekharan D et al. Tonsillectomy or adenotonsillectomy versus non-surgical management for obstructive sleep-disordered breathing in children. Cochrane Database Syst Rev 2015; 10: CD011165

[21] Gorman D, Ogston S, Hussain SS. Improvement in symptoms of obstructive sleep apnoea in children following tonsillectomy versus tonsillotomy: a systematic review and meta-analysis. Clin Otolaryngol 2017; 42: 275-282

[22] Geissler K, Bohne S, Siggel R et al. Preoperative serum pattern analysis to predict the outcome of tonsillectomy in adults with chronic tonsillitis. Eur Arch Otorhinolaryngol 2014; 271: 2803-2811

[23] Guntinas-Lichius O, Volk GF, Zaslansky R et al. The first postoperative day: prospective evaluation of pain in adult otorhinolaryngologic surgery. Clin J Pain 2014; 30: 978-986

[24] Guntinas-Lichius O, Geissler K, Preussler NP et al. Optimal Postoperative Pain Management After Tonsillectomy: An Unsolved Problem. Laryngorhinootologie 2016; 95: 15-23

[25] GmbH AlfaQuFiG. Tonsillenoperationen: Konzeptskizze für ein Qualitätssicherungsverfahren. In. Göttingen: AQUA - Institut für angewandte Qualitätsförderung und Forschung im Gesundheitswesen GmbH 2014

[26] Seethaler A, Rudack C, Spiekermann C. Structured literature review of patient-reported outcome (PRO) instruments in adult tonsillectomy or tonsillotomy. Health Qual Life Outcomes 2019; 17: 122

[27] McCulloch P, Nagendran M, Campbell WB et al. Strategies to reduce variation in the use of surgery. Lancet 2013; 382: 1130-1139

[28] Goldschmidt PG. The "Right Rate" of Tonsillectomy. JAMA Otolaryngol Head Neck Surg 2020. doi:10.1001/jamaoto. 2019.4206 [Epub ahead of print]

[29] Stasio SD, Yang C, Brietzke SE et al. Tonsillectomy versus tonsillotomy: a study of parental preference. Int J Ped Otorhinolaryngol 2015; 79: 359-362
[30] Brodsky JR, Cunningham MJ. Letter to the Editor regarding "Tonsillectomy versus Tonsillotomy: A Study of Parental Preference”. Int J Ped Otorhinolaryngol 2015; 79: 1788-1789

[31] Sutton RT, Pincock D, Baumgart DC et al. An overview of clinical decision support systems: benefits, risks, and strategies for success. NPJ Digit Med 2020; 3: 17

[32] SIGN. Management of sore throat and indications for tonsillectomy. A national clinical guideline. Edinburgh: SIGN publication. 2010

[33] Ware J Jr, Kosinski M, Keller SD. A 12-Item Short-Form Health Survey: construction of scales and preliminary tests of reliability and validity. Med Care 1996; 34: 220-233

[34] Ware JE jr, Gandek B. Overview of the SF-36 Health Survey and the International Quality of Life Assessment (IQOLA) Project. J Clin Epidemiol 1998; 51: 903-912

[35] Bullinger M, Kirchberger I. SF-36 Fragebogen zum Gesundheitszustand. Göttingen: Hogrefe; 1998

[36] Ravens-Sieberer U, Bullinger M. Assessing health-related quality of life in chronically ill children with the German KINDL: first psychometric and content analytical results. Qual Life Res 1998; 7: 399-407

[37] Ravens-Sieberer U, Erhart M, Rajmil L et al. Reliability, construct and criterion validity of the KIDSCREEN-10 score: a short measure for children and adolescents' well-being and health-related quality of life. Qual Life Res 2010; 19: 14871500

[38] Robinson K, Gatehouse S, Browning GG. Measuring patient benefit from otorhinolaryngological surgery and therapy. Ann Otol Rhinol Laryngol 1996; 105: 415-422

[39] Hendry J, Chin A, Swan IR et al. The Glasgow Benefit Inventory: a systematic review of the use and value of an otorhinolaryngological generic patient-recorded outcome measure. Clin Otolaryngol 2016; 41: 259-275

[40] Kubba H, Swan IR, Gatehouse S. The Glasgow Children's Benefit Inventory: a new instrument for assessing health-related benefit after an intervention. Ann Otol Rhinol Laryngol 2004; 113: 980-986

[41] Schwentner I, Schwentner C, Schmutzhard J et al. Validation of the German Glasgow children's benefit inventory. J Eval Clin Pract 2007; 13: 942-946

[42] Smith E, Wenzel S, Rettinger G et al. Quality of life in children with obstructive sleeping disorder after tonsillectomy, tonsillotomy or adenotomy. Laryngorhinootologie 2008; 87: 490497

[43] de Serres LM, Derkay C, Astley S et al. Measuring quality of life in children with obstructive sleep disorders. Arch Otolaryngol Head Neck Surg 2000; 126: 1423-1429

[44] Baumann I, Kucheida H, Blumenstock $G$ et al. Benefit from tonsillectomy in adult patients with chronic tonsillitis. Eur Arch Otorhinolaryngol 2006; 263: 556-559

[45] Stewart MG, Friedman EM, Sulek M et al. Validation of an outcomes instrument for tonsil and adenoid disease. Arch Otolaryngol Head Neck Surg 2001; 127: 29-35

[46] Steinbichler T, Bender B, Blassnigg E et al. Evaluation of a German version of the tonsil and adenoid health status instrument. J Otolaryngol Head Neck Surg 2014; 43: 41

[47] Skevas T, Klingmann C, Plinkert PK et al. Development and validation of the Tonsillectomy Outcome Inventory 14 . HNO 2012; 60: 801-806 
[48] Poller K, Volk GF, Wittekindt C et al. Verbesserung der Schmerztherapie nach Tonsillektomie bei Erwachsenen durch Schmerzmessung mit QUIPS („Qualitätsverbesserung in der postoperativen Schmerztherapie“). Laryngorhinootologie 2011; 90: 82-89

[49] Guntinas-Lichius O, Volk GF, Geissler K et al. Pain after pediatric otorhinolaryngologic surgery: a prospective multi-center trial. Eur Arch Otorhinolaryngol 2014; 271: 2049-2060

[50] Amrhein P, Di Dio D, Koitschev A et al. Tonsillektomie und Tonsillotomie im Wandel - Ist die Tonsillektomie bei Kindern noch zeitgemäß? Pädiatrie up2date 2019; 14: 215-224

[51] Gronau S, Fischer Y. Tonsillotomy. Laryngorhinootologie 2005; 84: 685-690; quiz 691-684

\section{Bibliografie}

DOI https://doi.org/10.1055/a-1148-2494

Laryngo-Rhino-Otol 2020; 99: 568-582

(c) Georg Thieme Verlag KG, Stuttgart · New York ISSN 0935-8943 


\section{Punkte sammeln auf CME.thieme.de}

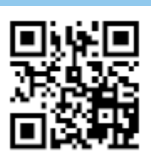

Diese Fortbildungseinheit ist in der Regel 12 Monate online für die Teilnahme verfügbar.

Den genauen Einsendeschluss finden Sie unter https://eref.thieme.de/CXEV7ZI.

Sollten Sie Fragen zur Online-Teilnahme haben, finden Sie unter https://cme.thieme.de/hilfe

eine ausführliche Anleitung. Wir wünschen viel Erfolg beim Beantworten

der Fragen!

Unter https://eref.thieme.de/CXEV7ZI oder über den QR-Code kommen Sie direkt zur Startseite des Wissenstests.

VNR 2760512020158724498

\section{Frage 1}

Welche Definition trifft zu?

A Bei einer Tonsillektomie findet eine vollständige intrakapsuläre Resektion der Tonsilla palatina statt.

B Unter Tonsillektomie wird die vollständige Entfernung der Tonsilla palatina verstanden.

C Unter Adenotonsillektomie wird die Entfernung der Tonsilla pharyngea und der Tonsilla lingualis zusammengefasst.

D Unter Tonsillotomie wird die Teilentfernung der Tonsilla pharyngea verstanden.

E Bei einer Tonsillektomie wird eine vollständige extrakapsuläre Resektion der Tonsilla pharyngea durchgeführt.

\section{Frage 2}

Welche Institution zählt nicht zu denjenigen, die sich in der Vergangenheit intensiv mit der Tonsillenchirurgie beschäftigt haben?
A Bertelsmann Stiftung
B AWMF
C IQWiG
D KV
E G-BA

\section{Frage 3}

Was zählt nicht zu den klassischen Indikationen zur Tonsillektomie?

A rezidivierende akute Tonsillitis

B mediane Halszyste

C Peritonsillarabszess

D Schlaf-Apnoe-Syndrom

E Tumorverdacht

\section{Frage 4}

Welche Aussage der AWMF-S2k-Leitlinie „Therapie entzündlicher Erkrankungen der Gaumenmandeln - Tonsillitis“ zur Tonsillektomie-Indikation trifft zu?

A Die Tonsillektomie ist eine therapeutische Option bei $\geq 6$ Episoden einer ärztlich diagnostizierten und mit Antibiotika therapierten eitrigen Tonsillitis.

B Die Tonsillektomie ist eine therapeutische Option bei < 3 Episoden einer ärztlich diagnostizierten und mit Antibiotika therapierten eitrigen Tonsillitis.

C Die Tonsillektomie ist bei 3-5 Episoden einer ärztlich diagnostizierten und mit Antibiotika therapierten eitrigen Tonsillitis keine mögliche Option, wenn sich innerhalb der nächsten 6 Monate weitere Episoden ereignen sollten und die Zahl 6 erreicht wird.

D Die Anzahl der Episoden einer ärztlich diagnostizierten und mit Antibiotika therapierten eitrigen Tonsillitis bezieht sich auf 24 Monate zum Zeitpunkt der Erstvorstellung.

E Als Episode kann auch eine ärztlich diagnostizierte und mit Antibiotika therapierte eitrige Pharyngolaryngitis zählen.

\section{Frage 5}

Welche Aussage zum Zweitmeinungsverfahren Tonsillenchirurgie trifft nicht zu?

A Vor einer Operation muss eine Bedenkzeit von 14 Tagen eingeräumt werden.

B Ein Zweitmeinungsverfahren muss dem Patienten offiziell angeboten werden.

C Es könnte im Fall von Komplikationen juristische Probleme durch die Unterlassung eines Zweitmeinungsverfahrens geben.

D Der die Zweitmeinung äußernde Arzt darf später nicht die Operation vornehmen.

E Die Zweitmeinung kann nur bei einem von der zuständigen Kassenärztlichen Vereinigung zugelassenen Zweitmeiner eingeholt werden.

- Weitere Fragen auf der folgenden Seite... 


\section{Punkte sammeln auf CME.thieme.de}

\section{Frage 6}

Welche Aussage zum IQWiG-Bericht trifft nicht zu?

A Zum Vergleich Tonsillotomie versus konservative Behandlung ließen sich weder relevante noch laufende Studien identifizieren, also war keine Aussage zu treffen.

B Generell habe die Tonsillotomie im Vergleich zur Tonsillektomie kurzfristige Vorteile für 2 Wochen: Es gebe Hinweise und geringe Anzeichen dafür, dass Schmerzen, Schluckstörungen und Schlafstörungen nach Tonsillotomie im Vergleich zur Tonsillektomie seltener auftreten.

C Auch für die patientenrelevanten Endpunkte wie postoperative Blutungen, Krankenhausaufenthaltsdauer, Hospitalisierung, gesundheitsbezogene Lebensqualität und sonstige unerwünschte Wirkungen habe sich ein Anhaltspunkt für einen höheren Nutzen der Tonsillotomie ergeben.

D Bei der Indikation rezidivierende akute Tonsillitis und auch bei der Indikation Tonsillenhyperplasie fehlen Langzeitdaten.

E Die Datenlage für den Endpunkt erneute Tonsillenoperation sei unzureichend gewesen.

\section{Frage 7}

Welche Aussage zur Tonsillotomie trifft zu?

A Die Tonsillotomie ist als Leistung der gesetzlichen Krankenkassen für die Behandlung einer symptomatischen und klinisch relevanten Tonsillenhyperplasie im Krankenhaus und in der ambulanten Medizin zugelassen.

B Die Tonsillotomie ist nur bei Patienten unter 18 Jahren indiziert.

C Eine Tonsillotomie muss zur besseren Versorgung der Patienten im Krankenhaus durchgeführt werden.

D Eine Tonsillotomie muss aus ökonomischen Gründen ambulant erfolgen.

E Im ambulanten Sektor werden Anforderungen für Operationen über den Katalog ambulant durchführbarer Operationen (AOPKatalog) geregelt. Im Moment steht noch die Festlegung aus, ob die Tonsillotomie als optional ambulant (Kategorie 1) oder obligat ambulant (Kategorie 2) eingeordnet wird.

\section{Frage 8}

Was ist nicht eine typische Komplikation oder Beschwerde nach Tonsillektomie?
A Nachblutung
B Schluckbeschwerden
C Ohrenschmerzen
D Foetor ex ore
E offenes Näseln

\section{Frage 9}

Was trifft zu? Im Jahr 2018 wurden in Deutschland ...

A etwa 80000 Tonsillektomien ohne Adenotomie unter stationären Bedingungen durchgeführt.

B etwa 30000 Tonsillektomien mit Adenotomie unter stationären Bedingungen durchgeführt.

C etwa 40000 Adenotomien unter stationären Bedingungen durchgeführt und damit gleichbleibend viele Adenotomien wie 2005.

D etwa 10000 Tonsillektomien ohne Adenotomie unter stationären Bedingungen durchgeführt.

E gleichbleibend viele Tonsillektomien unter stationären Bedingungen durchgeführt wie 2005.

\section{Frage 10}

Welche Aussage trifft zu?

A Aufgrund des Nachblutungsrisikos sollte die Tonsillotomie im Allgemeinen nur in Ausnahmesituationen bei Kindern durchgeführt werden.

B Die Tonsillenchirurgie hat aufgrund postoperativer Komplikationen den Status als Routineoperation in der HNO-Heilkunde verloren.

C Eine geografische Variabilität hinsichtlich der TonsillektomieOperationsraten wird in Deutschland nicht beobachtet.

D Bei OSAS kann sowohl eine Tonsillotomie als auch eine Tonsillektomie durchgeführt werden.

E Die NATTINA-Studie untersucht die Effektivität einer Tonsillotomie und Tonsillektomie bei rezidivierender akuter Tonsillitis. 